\section{Quality traits of Ciauscolo salami from meat of pigs fed rosemary extract enriched diet}

\author{
David Ranucci, ${ }^{1}$ Raffaella Branciari, ${ }^{1}$ \\ Gabriele Acuti, ${ }^{2}$ Giacinto Della Casa, ${ }^{3}$ \\ Massimo Trabalza-Marinucci, ${ }^{2}$ \\ Dino Miraglia ${ }^{1}$
}

1Dipartimento di Scienze Biopatologiche ed Igiene delle Produzioni Animali e

Alimentari, Università di Perugia;

2Dipartimento di Patologia, Diagnostica e

Clinica Veterinaria, Università di Perugia;

${ }^{3}$ Unità di ricerca per la suinicoltura -

CRA, Modena, Italy

\section{Abstract}

The microbiological, chemical-physical and organoleptic characteristics of four batches of Ciauscolo salami, two made from meat of pigs fed diet integrated with $0.2 \%$ of rosemary extract (RS) and two controls (CSs), were considered. Three samples for each batch were in double analyzed for total bacterial count at $30^{\circ} \mathrm{C}$, enumeration of lactococci, lactobacilli, staphylococcus coagulase positive, enterococci, Enterobacteriaceae, and isolation of Salmonella spp. and Listeria monocytogenes, after filling and at 7 and 20 days of ripening. On the same samples, measurement of $\mathrm{pH}$ (pHmeter MP120; Mettler-Toledo Spa, Schwerzenbach, Switzerland), activity water (aw) (Hygroscope BT-RS1 Rotronic; PBI International, Milan, Italy) and CIE $\mathrm{L}^{*} \mathrm{a}^{*} \mathrm{~b}^{*}$ colour (Chromameter Minolta C400; Minolta Ltd., Osaka, Japan) were performed. Proximal composition, $\mathrm{NaCl}$ content (AOAC, 1990) thiobarbituric reactive substances (TBARs) and panel test (ISO 8586-1:1993 and ISO 8586) were performed only on samples obtained at the end of the ripening time.

No difference in proximal composition, $\mathrm{pH}$, $\mathrm{a}_{\mathrm{w}}$ values and microbial counts between CS and RS samples were observed along the whole production period. Colour analyses reveal higher $\mathrm{a}^{*}$ values in $\mathrm{RS}(10.79$ vs $9.68, \mathrm{P}<0.05)$. Higher TBARs mean value was recorded in CS at the end of ripening (1.12 vs $0.91 \mathrm{mg}$ MDA/100g, $\mathrm{P}<0.01$ ). Even if no statistical differences were recorded in all the parameters considered in sensory evaluation, the overall acceptance of RS samples tended to be higher than CS.

\section{Introduzione}

Sin dai tempi antichi alcune erbe aromatiche e spezie sono state aggiunte ai diversi tipi di cibo per migliorarne il sapore e le proprietà organolettiche. Esse hanno acquisito recentemente un grande interesse nell'emergente industria degli alimenti funzionali in quanto possono essere inserite nel cibo come farmaco naturale, essendo correntemente impiegate nella terapia e profilassi di numerose malattie in tutto il mondo (Diploc et al., 1998). Il loro utilizzo in fitoterapia è per lo più legato alla presenza di numerose molecole secondarie, in particolare oli essenziali, che sono in grado di esplicare azione antimicrobica, spasmolitica, carminativa, epatoprotettiva, antivirale e antitumorale (Bruneton 1999; Darsham e Doreswamy, 2004; Pan et al., 2010; Herrmann et al., 2011). Proprio l'attività antimicrobica è di particolare interesse per i veterinari igienisti, come confermano numerosi studi volti a valutare le capacità inibitorie in vitro di differenti essenze nei confronti di batteri e miceti patogeni e alteranti (Ouattara et al., 1997; Burt, 2004; Holley e Patel, 2005). Altro interesse dell'industria alimentare è legato all'azione antiossidante esplicata da molti composti isolati da erbe che li rendono utilizzabili come antiossidanti naturali e proposti come potenziali sostituti di quelli sintetici (Parejo et al., 2002; Bozin et al., 2007). Tra le varie erbe aromatiche, il rosmarino, per la presenza di numerosi componenti bioattivi (Pintore et al., 2002; Ibanez et al., 2003), è stato oggetto di studio per valutare le sue capacità antiossidanti e antimicrobiche sia in vitro (Ouattara et al., 1997; Cavero et al., 2005; Bozin et al., 2007; Dragoni et al., 2009) che aggiungendolo direttamente al prodotto finito, o comunque durante la sua lavorazione (Sebranek et al., 2005; Martinez et al., 2006; Georgantelis et al., 2007). La valutazione della quantità da aggiungere sarebbe legata, per l'estrinsecarsi dell'effetto voluto, anche alla possibilità dei composti di interagire con le differenti componenti dell'alimento (Gutierrez et al., 2008; Tajkarimi et al., 2010). L'effetto sarà comunque proporzionato al quantitativo di sostanze aggiunte all'alimento, anche se, all'aumentare del dosaggio, si corre il rischio di indurre alterazioni organolettiche, con conseguente minor accettabilità del prodotto da parte del consumatore (Gutierrez et al., 2008). Recentemente è stata valutata la possibilità di far pervenire i composti bioattivi agli alimenti direttamente tramite l'alimentazione somministrata agli animali (Monino et al., 2008; Haak et al., 2008; Nieto et al., 2011). Quali siano gli effetti sugli alimenti derivati è ancora da dimostrare in particolare nel suino, dove alcuni prodotti a base di carne subiscono fondamentali processi fermentativi di origine batterica che non devono
Correspondence: David Ranucci, Dipartimento di Scienze Biopatologiche ed Igiene delle Produzioni Animali e Alimentari, Università di Perugia, Italy.

Tel. +39.75.5857932 - Fax: +39.75.5857927

E-mail: david.ranucci@unipg.it

Key words: Rosemary extract, Dry fermented sausage, Food microbiology, Lipid oxidation, Sensory anaysis.

Conflict of interests: the authors declare no potential conflict of interests.

Acknowledgments: the Authors are thankful to Alessandro Filippini and Dario Zanichelli (Phenbiox S.r.l, Bologna) for their co-operation in preparing rosemary extract and Corte Marchigiana S.r.l (Camerata Picena, Italy) for salami production.

Funding: the current research has been carried out within the project Industria 2015. Alimentazione funzionale nella filiera agro-alimentare (suino, pollo e coniglio) per il miglioramento del benessere animale e per il trasferimento di componenti nutrizionali funzionali al miglioramento della salute dell'uomo [i.e. Functional diet in agricultural food chain (pork, chicken and rabbit) to ameliorate animal welfare and transfer functional nutritional components for people's better health] promoted by the Italian Welfare Ministry.

Received for publication: 15 January 2013.

Revision received: 5 March 2013.

Accepted for publication: 21 March 2013

This work is licensed under a Creative Commons Attribution 3.0 License (by-nc 3.0).

CCopyright D. Ranucci et al., 2013

Licensee PAGEPress, Italy

Italian Journal of Food Safety 2013; 2:e16

doi:10.4081/ijfs.2013.e16

essere inibiti. Scarsi sono ad oggi gli studi sull'effetto degli estratti vegetali su microrganismi tecnologici, in particolare quelli impiegati come colture starter (Ouattara et al., 1997; Seydim e Sarikus, 2006).

Scopo del presente lavoro è stato quindi quello di valutare le caratteristiche microbiologiche, chimico-fisiche e organolettiche di salame Ciauscolo prodotto con carne di suini alimentati con una dieta integrata con estratto di rosmarino.

\section{Materiali e Metodi}

Il prodotto scelto per la sperimentazione è stato il salame Ciauscolo, insaccato tipico dell'appennino umbro-marchigiano (Rea et al., 2003) e inserito nella lista dei salumi con 
Indicazione Geografica Protetta (IGP). Sono stati prodotti 4 lotti di salame, 2 con carne proveniente da suini controllo (SC) e 2 con carne di suini alimentati con integrazione di estratto di rosmarino (SR). Gli animali, suini pesanti di incrocio Duroc Italiana $x$ Large White Italiana, sono stati allevati presso l'allevamento del CRA (Unità di Ricerca per la Suinicoltura) dal peso vivo di $35 \mathrm{~kg}$ fino a quello di $170 \mathrm{~kg}$ circa. I due gruppi sperimentali, ognuno costituito da 16 soggetti, sono stati alimentati in maniera differenziale con la dieta SC (mangime controllo) oppure la dieta SR (mangime addizionato di estratto acquoso di rosmarino Phenbiox in ragione dello $0,2 \%$ del tal quale) per l'intera prova. Entrambi i mangimi contenevano lo $0,5 \%$ sul tal quale di acido linoleico coniugato nella fase di finissaggio a partire dai $120 \mathrm{~kg}$ di peso vivo. I suini sono stati alimentati in due pasti giornalieri con somministrazione dell'alimento in forma umida con rapporto acqua:mangime di 3:1.

La produzione è avvenuta in un impianto industriale, secondo quanto previsto dal disciplinare di produzione IGP con aggiunta di additivi (E301 - ascorbato di sodio: 0,060 kg/q di carne; E251 - nitrato di sodio: $0,020 \mathrm{~kg} / \mathrm{q}$ di carne). Non è stato previsto l'impiego di microrganismi starter in entrambi i prodotti. Durante le fasi di lavorazione, a tempi stabiliti [tempo 0 (T0): insacco; tempo 1 (T1): 7 giorni - dopo asciugatura; tempo 2 (T2): 20 giorni prodotto finito], sono stati prelevati 3 campioni per lotto da sottoporre ad analisi. I campioni, posti in contenitori refrigerati, sono stati trasferiti presso il laboratorio dove si è provveduto a svolgere nella stessa giornata del prelievo le seguenti analisi microbiologiche e chimico-fisiche (in doppio su 3 salami per lotto a tempi differenti di stagionatura): carica batterica totale (CBT) a $30^{\circ} \mathrm{C}$; numerazione lattococchi a $30^{\circ} \mathrm{C}$ per $48 \mathrm{~h}$ (M17 Agar; Biokar Diagnostics, Beauvais, Francia); numerazione lattobacilli a $37^{\circ} \mathrm{C}$ per $72 \mathrm{~h}$ in anaerobiosi (MRS Agar acidificato; 0xoid, Basingstoke, UK); numerazione stafilococchi coagulasi positivi (UNI EN ISO 6888-1:2004; UNI, 2004b)
; numerazione enterococchi (IS0 7899-2:2003; UNI, 2003), numerazione Enterobacteriaceae (ISO 21528-2:2004; ISO, 2004); ricerca di Salmonella spp. (ISO 6579:2004; UNI, 2004a) e Listeria monocytogenes (ISO 11290-1:2005; UNI, 2005); determinazione del pH mediante pHMetro ad infissione (MP120; Mettler-Toledo Spa, Schwerzenbach, Svizzera), determinazione dell'acqua libera [activity water $\left(\mathrm{a}_{\mathrm{w}}\right)$ ] mediante igroscopio (BT-RS1 Hygroscope Rotronic, PBI International, Milano, Italia); e determinazione del colore con sistema International Commission on Illumination (CIE) $\mathrm{L}^{*} \mathrm{a}^{*} \mathrm{~b}^{*}$ (CIE, 1986) mediante colorimentro (Minolta C400; Minolta Ltd., Osaka, Giappone).

Solo sui campioni di prodotto finito si è provveduto alla valutazione della composizione chimica centesimale (AOAC, 1990), del contenuto in cloruro di sodio (AOAC, 1990) e delle sostanze reattive all'acido tiobarbiturico (TBARs; Tarladgis et al., 1960).

Le valutazione sensoriali sono state eseguite da 6 assaggiatori addestrati dell'Università degli Studi di Perugia secondo i criteri descritti dalla norma ISO 8586-1:1993 (ISO, 1993) e ISO 8586-2:1994 (ISO, 1994) e dalla quantitative descriptive analysis (QDA) (Lawless $\mathrm{e}$ Heymann, 1999; Stone e Sidel, 2004). Sono state organizzate alcune sessioni propedeutiche al test vero e proprio, per concordare il lessico sensoriale e le definizioni degli attributi e per calibrare il panel con campioni di riferimento per l'utilizzo delle scale quantitative (Ruiz Pérez-Cacho et al., 2005). Le prove di assaggio sono state condotte su fette di salame di $2 \mathrm{~mm}$ di spessore, i campioni, una volta portati alla temperatura di $20 \pm 1^{\circ} \mathrm{C}$, sono stati sottoposti a valutazione senza fornire ai giudici informazioni sulla loro identità. Tutti i campioni sono stati serviti in contenitori di polipropilene, con coperchio e codificati con triplette di numeri. Gli assaggiatori sono stati istruiti a sciacquare la bocca con acqua a basso contenuto di sali prima di ogni valutazione e a masticare un cracker per eliminare i sapori residui. I diversi parametri sono stati valutati in una scala non strutturata con valori da 0 a 9 .

L'elaborazione dei dati è stata effettuata mediante test statistici parametrici utilizzando unpaired t-test del programma Statview (SAS Institute inc., Cary, NC, USA), confrontando, a parità di tempo di produzione, i dati dei singoli parametri presi in considerazione nelle due tipologie di salame. Il livello di significatività è stato fissato a valori di $\mathrm{P} \leq 0,05$.

\section{Risultati}

L'andamento dei valori medi di $\mathrm{pH}$ e $\mathrm{a}_{\mathrm{w}}$ nei due gruppi di salami, nei tempi considerati, è evidenziato in Tabella 1. Per quanto riguarda il colore, non è stata riscontrata un differenza significativa nei valori medi di $\mathrm{L}^{*}$ sia a T1 (SC: $61,77 \pm 2,59$ vs SR $59,92 \pm 1,49$ ) che a T2 (SC: $61,00 \pm 0,64$ vs SR: $60,47 \pm 1,74)$ e di b* sia a T1 (SC: $8,89 \pm 1,57$ vs SR: $9,26 \pm 0,95)$, che a T2 (SC:7,68 $\pm 0,64$ vs SR:8,65 $\pm 0,84$ ). È stata invece notata una differenza significativa $(\mathrm{p}<0,05)$ nei valori di $\mathrm{a}^{*}$ sia a T1 (SC: $10,52 \pm 1,39$ vs SR: $12,22 \pm 1,27$ ) che a T2 (SC: $9,68 \pm 0,34$ vs SR: $10,79 \pm 1,12$ ). Per quanto riguarda le caratteristiche microbiologiche dei salami (Tabella 1), non sono emerse differenze sostanziali tra i lotti SC e SR per le varie determinazioni microbiologiche. Già a partire dall'impasto non è stata rilevata la presenza di Salmonella spp., stafilococchi coagulasi positivi e Listeria monocytogenes. Anche per quanto riguarda la composizione chimica centesimale e la concentrazione di $\mathrm{NaCl}$, i campioni SC e SR a fine stagionatura non hanno evidenziato differenze significative, mentre i valori di TBARs sono risultati maggiori nei $\mathrm{SC}(\mathrm{P}<0,01)$ (Tabella 2). I dati relativi alla valutazione sensoriale sono riportati nella Figura 1. Nonostante non siano emerse differenze significative tra i due gruppi in esame per tutti i descrittori considerati, i lotti SR sono risultati tendenzialmente più consistenti, meno umidi, con minor odore e sapore di rancido e grasso e con un giudizio complessivo migliore.

Tabella 1. Andamento del $\mathrm{pH}$, determinazione dell'acqua libera e delle conte microbiologiche nei salami controllo e rosmarino a differenti tempi di produzione.

\begin{tabular}{|c|c|c|c|c|c|c|c|c|c|c|}
\hline Salami & $\begin{array}{l}\text { Campioni } \\
\text { (n) }\end{array}$ & Tempo & $\mathrm{pH}^{*}$ & $a w^{*}$ & $\begin{array}{c}\text { CBT * } \\
(\log \mathrm{cfu} / \mathrm{g})\end{array}$ & $\begin{array}{l}\text { Lattococchi } \\
(\log \mathrm{cfu} / \mathrm{g}) *\end{array}$ & $\begin{array}{l}\text { Lattobacilli } \\
\text { (Log cfu/g)* }\end{array}$ & $\begin{array}{l}\text { Stafilococchi } \\
(\log \mathrm{cfu} / \mathrm{g})^{*}\end{array}$ & $\begin{array}{l}\text { Enterococchi } \\
(\log \mathrm{cfu} / \mathrm{g})^{*}\end{array}$ & $\begin{array}{l}\text { Enterobatteri } \\
(\log \mathrm{cfu} / \mathrm{g}) *\end{array}$ \\
\hline $\mathrm{SC}$ & 6 & T0 & $5,80 \pm 0,10$ & $0,955 \pm 0,002$ & $5,59 \pm 0,35$ & $5,19 \pm 0,25$ & $5,15 \pm 0,31$ & $4,07 \pm 0,27$ & $3,34 \pm 0,27$ & $3,88 \pm 0,30$ \\
\hline SR & 6 & T0 & $5,78 \pm 0,08$ & $0,954 \pm 0,001$ & $5,10 \pm 0,18$ & $4,70 \pm 0,47$ & $4,02 \pm 0,32$ & $3,78 \pm 0,49$ & $3,18 \pm 0,23$ & $3,30 \pm 0,05$ \\
\hline $\mathrm{SC}$ & 6 & $\mathrm{~T} 1$ & $5,15 \pm 0,04$ & $0,940 \pm 0,002$ & $8,63 \pm 0,10$ & $7,07 \pm 0,03$ & $8,65 \pm 0,10$ & $5,11 \pm 0,13$ & $4,13 \pm 0,05$ & $3,00 \pm 0,07$ \\
\hline SR & 6 & $\mathrm{Tl}$ & $5,17 \pm 0,03$ & $0,937 \pm 0,001$ & $8,89 \pm 0,08$ & $7,21 \pm 0,38$ & $8,84 \pm 0,06$ & $5,74 \pm 0,40$ & $4,74 \pm 0,21$ & $2,60 \pm 0,19$ \\
\hline $\mathrm{SC}$ & 6 & $\mathrm{~T} 2$ & $5,13 \pm 0,02$ & $0,918 \pm 0,002$ & $8,58 \pm 0,29$ & $5,47 \pm 0,13$ & $8,61 \pm 0,05$ & $4,31 \pm 0,32$ & $3,97 \pm 0,08$ & $<2,00$ \\
\hline SR & 6 & $\mathrm{~T} 2$ & $5,13 \pm 0,04$ & $0,918 \pm 0,005$ & $8,30 \pm 0,31$ & $5,93 \pm 0,50$ & $8,63 \pm 0,08$ & $4,68 \pm 0,23$ & $4,87 \pm 0,58$ & $<2,00$ \\
\hline
\end{tabular}

$\mathrm{a}_{w}$, activity water (determinazione dell'acqua libera); CBT, carica batterica totale; SC, salami controllo; SR, salami rosmarino; T0, insacco; T1, 7 giorni; T2, 20 giorni. *Le conte microbiologiche sono espresse in valore medio \pm deviazione standard. 
Tabella 2. Composizione chimica centesimale, concentrazione di $\mathrm{NaCl}$ e sostanze reattive all'acido tiobarbiturico nei salami controllo e rosmarino (valore medio \pm deviazione standard).

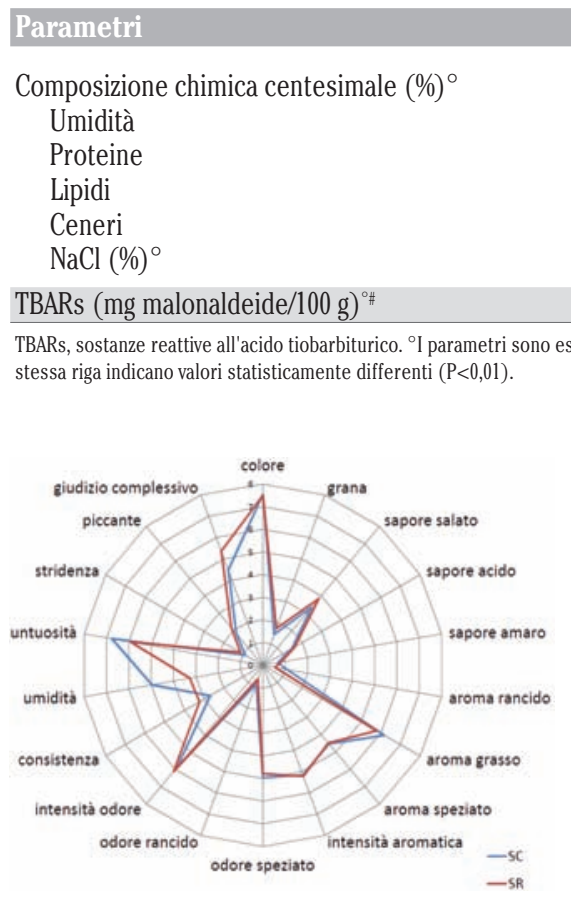

Figura 1. Risultati della valutazione sensoriale su salami controllo (SC) e rosmarino (SR).

\section{Discussione}

I dati relativi alle caratteristiche chimicofisiche e centesimali dei prodotti in sperimentazione sono sovrapponibili a quelli evidenziati da altri autori in salame Ciauscolo (Rea et al., 2005; Aquilanti et al., 2007; Trani et al., 2010). Si conferma quindi la caratteristica alta percentuale di grasso del prodotto, il pH acido e l'a $a_{w}$ inferiore a 0,92 già a 20 giorni di stagionatura. La dieta con rosmarino non ha permesso di evidenziare effetti inibitori nei confronti dei batteri presenti nell'impasto. Circa l'effetto degli estratti di rosmarino su batteri comunemente impiegati nei salami, i dati presenti in bibliografia sono spesso contrastanti. Alcune sperimentazioni in vitro hanno evidenziato effetti del rosmarino nei confronti di Lactobacillus sakei e Lactobacillus curvatus (Ouattara et al., 1997), ma non nei confronti di Lactobacillus plantarum (Seydim and Sarikus, 2006). L'aggiunta di estratto di rosmarino in salsiccia fresca non ha evidenziato riduzione della conta dei batteri lattici rispetto a campioni controllo (Gerogantelis et al., 2007), mentre ha inibito lo sviluppo di $L$. curvatus in salame (Verluyten et al., 2004). Inoltre, l'eventuale effetto antibatterico dovuto alla somministrazione dell'estratto agli animali non è al momento dimostrato, anche se alcuni autori riportano una riduzione della CBT in carcasse di agnelli alimentati con latte di pecore a cui era stato somministrato estratto di rosmarino (Banon et al., 2012). Il mancato riscontro di patogeni già nell'impasto in entrambi i gruppi in sperimentazione non ha permesso di valutare il possibile effetto della dieta su tali specie microbiche. Per quanto riguarda l'effetto antiossidante, il riscontro di valori di a* più elevati e di TBARs più bassi permette di affermare che la somministrazione di estratto di rosmarino con la dieta è in grado di limitare gli inevitabili processi ossidativi delle carni che avvengono durante la fermentazione in tutte le tipologie di insaccati stagionati (Chizzolini et al., 1998). Il riscontro di un maggior colore rosso delle carni è confermato da altri lavori effettuati aggiungendo composti attivi di rosmarino in salsiccia non stagionata di suino (Sebranek et al., 2005; Martinez et al., 2006). Relativamente ai TBARs, i dati riscontrati confermano quanto riportato da altri autori su carne fresca di suini alimentati con diete integrate da estratto di rosmarino (Haack et al., 2008). La presenza di sostanze antiossidanti, originarie del rosmarino, pervenute a livello muscolare sembra quindi inibire l'ossidazione sia dei lipidi che della mioglobina aumentando, quindi, la stabilità del colore (Gatellier et al., 2001). Per quanto riguarda le caratteristiche organolettiche, l'impiego del rosmarino nella dieta sembrerebbe non influenzare la percezione delle componenti visive, olfattive e gustative tipiche del Ciauscolo. Sotto questo aspetto non esisterebbero delle controindicazioni all'impiego di estratto di rosmarino nella dieta dei suini.

\section{Conclusioni}

In conclusione, l'impiego di rosmarino nella dieta può indurre effetti antiossidanti senza andare ad alterare la fermentazione dei prodotti, anche se particolare attenzione andrà rivolta a possibili correlazioni tra presenza di composti attivi del rosmarino, qualità sensoriale del salame e accettabilità del prodotto da parte del consumatore. Ulteriori studi saranno necessari per valutare la reale concentrazione dei composti bioattivi nella carne degli animali alimentati con rosmarino e se queste molecole possano esplicare un effetto antibatterico nei confronti di microrganismi patogeni.

\section{Bibliografia}

Aquilanti L, Santarelli S, Silvestri G, Osimani A, Petruzzelli A, Clementi F, 2007. The microbial ecology of a typical Italian salami during its natural fermentation. Int $\mathrm{J}$ Food Microbiol 120:136-45.

AOAC, 1990. Official Methods of Analysis. 15th ed. Association of Official Analytical Chemists ed., Washington, DC, USA.

Banon S, Mendez L, Almela E, 2012. Effects of dietary rosemary extract on lamb spoilage under retail display conditions. Meat Sci 90:579-83.

Bozin B, Mimica-Dukic N, Samojlik I, Jovin E, 2007. Antimicrobial and antioxidant properties of rosemary and sage (Rosmarinus officinalis L. and Salvia officinalis L., Lamiaceae) essential oils. J Agr Food Chem 55:7879-85.

Bruneton J, 1999. Pharmacology, phytochemistry, medical plants. 2nd ed. Intercept Ltd., London, UK.

Burt S, 2004. Essential oils: their antibacterial properties and potential applications in foods - a review. Int $\mathrm{J}$ Food Microbiol 94:223-53.

Cavero S, Jaime L, Martin-Alvarez PJ, Senorans FJ, Reglero G, Ibanez E, 2005. In vitro antioxidant analysis of supercritical fluid extracts from rosemary (Rosmarinus officinalis L.). Eur Food Res Technol 221:478-86.

Chizzolini R, Novelli E, Zanardi E, 1998. 0xidation in traditional Mediterranean meat products. Meat Sci 49:S87-99.

CIE, 1986. Colorimetry 15.2. Commission Internationale de l'Eclairage Publ., Vienna, Austria.

Darsham S, Doreswamy R, 2004. Patented antiinflammatory plant drug development from traditional medicine. Phytother Res 18:343-57.

Diploc AT, Charleux JL, Crozier-Willi G, Kock FJ, Rice-Evans C, Roberfroid M, Stahl W, Vina-Ribes J, 1998. Functional food science and defence against reactive oxidative species. Brit J Nutr 80:S77-112.

Dragoni I, Vallone L, 2009. Antifungal activity of rosemary essential oil against foodstuffs fungi. Rivista AIVI 4:17-20.

Gatellier P, Hamelin C, Durand Y, Renerre M, 2001. Effect of a dietary vitamin E supplementation on colour stability and lipid oxidation of air- and modified atmosphere- 
packaged beef. Meat Sci 59:133-40.

Georgantelis D, Ambrosiadis I, Katikou P, Blekas G, Georgakis SA, 2007. Effect of rosemaryextract, chitosan and a-tocopherol on microbiological parameters and lipid oxidation of fresh pork sausages stored at $4^{\circ} \mathrm{C}$. Meat Sci 76:172-81.

Gutierrez J, Barry-Ryan C, Bourke P, 2008. The antimicrobial efficacy of plant essential oil combinations and interactions with food ingredients. Int J Food Microbiol 124:91-7.

Haak L, Raes K, Van Dyck S, De Smet S, 2008. Effect of dietary rosemary and a-tocopheryl acetate on the oxidative stability of raw and cooked pork following oxidized linseed oil administration. Meat Sci 78:239-47.

Herrmann F, Romero MR, Blazquez AG, Kaufmann D, Ashour ML, Kahl S, Marin JJG, Efferth T, Wink M, 2011. Diversity of pharmacological properties in Chinese and European medicinal plants: cytotoxicity, antiviral and antitrypanosomal screening of 82 herbal drugs. Diversity 3:547-80.

Holley RA, Patel D, 2005. Improvement in shelflife and safety of perishable foods by plant essential oils and smoke antimicrobials. Food Microbiol 22:273-92.

Ibanez E, Kubatova A, Senorans FJ, Cavero S, Reglero G, Hawthorne SB, 2003. Subcritical water extraction of antioxidant compounds from rosemary plants. J Agr Food Chem 51:375-82.

ISO, 1993. Sensory analysis. General guidance for the selection, training and monitoring of assessors. Part 1: selected assessors. Norma ISO 8586-1:1993. Organizzazione internazionale per la normazione ed., Ginevra, Svizzera.

ISO, 1994. Sensory analysis. General guidance for the selection, training and monitoring of assessors. Part 2: experts. Norma ISO 8586-2:1994. Organizzazione internazionale per la normazione ed., Ginevra, Svizzera.

ISO, 2004. Microbiology of food and animal feeding stuffs. Horizontal methods for the detection and enumeration of Enterobacteriaceae. Part 2: colony-count method. Norma ISO 21528-2:2004. Organizzazione internazionale per la normazione ed., Ginevra, Svizzera.

Lawless HT, Heymann H, 1999. Sensory evaluation of food: principles and practices. 1st ed. Chapman \& Hall, New York, NY, USA.

Martinez L, Cilla I, Beltran JA, Roncales P,
2006. Combined effect of modified atmosphere packaging and addition of rosemary (Rosmarinus officinalis), ascorbic acid, red beet root (Beta vulgaris), and sodium lactate and their mixtures on the stability of fresh pork sausages. J Agr Food Chem 54:4674-80.

Monino I, Martinez C, Sotomayor JA, Lafuente A, Jordan MJ, 2008. Polyphenolic transmission to Segureño lamb meat from ewes' diet supplemented with the distillate from rosemary (Rosmarinus officinalis) leaves. J Agr Food Chem 56:3363-7.

Nieto G, Estrada M, Jordan MJ, Garrido MD, Banon S, 2011. Effects in ewe diet of rosemary by-product on lipid oxidation and the eating quality of cooked lamb under retail display conditions. Food Chem 124:1423-9.

Ouattara B, Simard RE, Holley AR, Piette GJP, Bégin A, 1997. Antibacterial activity of selected fatty acids and essential oils against six meat spoilage organisms. Int J Food Microbiol 37:155-62.

Pan L, Chai H, Douglas Kinghorn A, 2010. The continuing search for antitumor agents from higher plants. Phytochem Lett 3:1-8.

Parejo I, Viladomat F, Bastida J, Rosas-Romero A, Flerlage N, Burillo J, Codina C, 2002. Comparison between the radical scavenging activity and antioxidant activity of six distilled and nondistilled Mediterranean herbs and aromatic plants. J Agr Food Chem 50:6882-90.

Pintore G, Usai M, Bradesi P, Juliano C, Boatto G, Tomi F, Chessa M, Cerri R, Casanova J, 2002. Chemical composition and antimicrobial activity of Rosmarinus officinalis L. oils from Sardinia and Corsica. Flavour Frag J 17:15-9.

Rea S, Cecchini S, Stocchi R, Loschi AR, Ricciutelli M, 2005. Chemical and physical traits and evaluation of biogenic amine content by RP-HPLC technique in Ciauscolo salami. Ind Aliment-Italy 44:3845.

Rea S, Pacifici L, Stocchi R, Loschi AR, 2003. Storia - caratteristiche e tecnologia di produzione del Ciauscolo. Ind Aliment-Italy 42:371-7.

Ruiz Pérez-Chaco MP, Galan-Soldevilla H, Léon Crespo F, Molina Recio G, 2005. Determination of the sensory attributes of Spanish dry-cured sausage. Meat Sci 71:620-33.

Sebranek JG, Sewalt VJH, Robbins KL, Houser
TA, 2005. Comparison of a natural rosemary extract and BHA/BHT for relative antioxidant effectiveness in pork sausage. Meat Sci 69:289-96.

Seydim AC, Saricus G, 2006. Antimicrobial activity of whey protein based edible films incorporated with oregano, rosemary and garlic essential oils. Food Res Int 39:63944.

Stone H, Sidel JL, 2004. Sensory evaluation practices. 3rd ed. Elsevier Academic Press, London, UK.

Tajkarimi MM, Ibrahim SA, Cliver D0, 2010. Antimicrobial herb and spice compounds in food. Food Control 21:1199-218.

Tarladgis BG, Watts BM, Younatham MT, 1960. A distillation method for the quantitative determination of malonaldhyde in rancid foods. J Am Oil Chem Soc 37:44-8.

Trani A, Gambacorta G, Loizzo P, Alviti G, Schena A, Faccia M, Aquilanti L, Santarelli S, Di Luccia A, 2010. Biochemical traits of Ciauscolo, a spreadable typical Italian drycured sausage. J Food Sci 75:C514-24.

UNI, 2003. Norma UNI EN ISO 7899-2:2003. Qualità dell acqua. Ricerca ed enumerazione di enterococchi intestinali. Metodo di filtrazione su membrana. Ente Nazionale Italiano di Unificazione ed., Milano, Italia.

UNI, 2004a. Norma UNI EN ISO 6579:2004. Microbiologia di alimenti e mangimi per animali. Metodo orizzontale per la ricerca di Salmonella spp. Ente Nazionale Italiano di Unificazione ed., Milano, Italia.

UNI, 2004b. Norma UNI EN ISO 6888-1:2004. Microbiologia di alimenti e mangimi per animali. Metodo orizzontale per la conta di stafilococchi coagulasi-positivi (Staphy lococcus aureus e altre specie). Tecnica che utilizza il terreno agar Baird-Parker. Ente Nazionale Italiano di Unificazione ed., Milano, Italia.

UNI, 2005. Norma UNI EN ISO 11290-1:2005. Microbiologia di alimenti e mangimi per animali. Metodo orizzontale per la ricerca e la conta di Listeria monocytogenes. Parte 1: metodo per la ricerca. Ente Nazionale Italiano di Unificazione ed., Milano, Italia.

Verluyten J, Leroi F, de Vuyst L, 2004. Effects of different spices used in production of fermented sausages on growth of and curvacin A production by Lactobacillus curvatus LTH 1174. J Appl Microbiol 70:4807-13. 